\title{
NON-SYNDROMIC UNILATERALSUPPLEMENTAL MAXILLARY CENTRAL INCISOR: A CASE REPORT
}

\author{
Pradeep Tangade ${ }^{1}$, Manu Batra ${ }^{1}$, Ravishankar TL ${ }^{1}$, Amit Tirth ${ }^{1}$, Sumit Pal $^{1}$
}

\begin{abstract}
BACKGROUND: Supernumerary teeth are developmental anomaly. There are various theories explaining their occurrence. Maxilla is more commonly affected and males have higher predilection than females for having supernumerary teeth.

CASE DETAILS: The present case is presented with three permanent central incisors, all of them with normal morphology. On clinical and radiographic examination, it was identified as a case of nonsyndromic unilateral left supplemental maxillary central incisor. Treatment plan involved extraction of the supplemental tooth followed by orthodontic treatment to correct the malalignment of the teeth.

CONCLUSION: The presence of supplemental teeth of normal morphology is quite a rare condition and are commonly associated with various syndromes but, in rare cases, they also exists as non-syndromic forms. Supernumerary teeth can cause aesthetic or functional problems, especially when situated in the maxillary anterior region. Complications related to the phenomenon can be minimized by early detection and by carrying out a comprehensive treatment protocol.

KEYWORDS: supplemental, central incisor
\end{abstract}

\section{INTRODUCTION}

Supernumerary teeth may be defined as any teeth or tooth substance in excess of the usual configuration of twenty deciduous, and thirty-two permanent teeth (1). It can also be seen along with certain missing teeth in dentition and is also associated with certain syndromes.

Supernumerary teeth can be classified on the basis of position or form. Positional variations include mesiodens, paramolars, distomolars and parapremolars. Variations in form consist of conical types, tuberculate types, supplemental teeth and odontomes (2). Supernumerary teeth may vary from a simple odontome, through a conical or tuberculate tooth to a supplemental tooth which closely resembles a normal tooth. One or two supernumerary teeth most commonly involve the anterior maxilla, followed by the mandibular premolar region (2). The most common site affected in case of multiple supernumerary teeth is the mandibular premolar region (3).
Teeth involved in order of decreasing frequency according to Luten are-upper lateral incisors (50 per cent), mesiodens (36 per cent), upper central incisors (11 per cent), followed by bicuspids ( 3 per cent) (4). Supernumerary teeth are more common to occur in the maxilla 8.2 to 10 times as frequently as in the mandible and most commonly affect the premaxilla (5) with males being more commonly affected indicating sexual dimorphism $(4,6)$. Hogstrum and Andersson (7) are also reported a 2:1 ratio of sex distribution.

The aetiology behind supernumerary teeth is unclear. Some of the theories which have been put up by various researchers include atavism i.e. reversion to a more primitive type of dentition. According to another theory, continued proliferation of remnants of the dental lamina may produce a "third dentition". According to the dichotomy theory, which seems the most credible, the tooth bud splits into two parts of equal or unequal size. This results into two teeth of equal size or one normal and one abnormal tooth, respectively. Gemination, which is assumed to be

\footnotetext{
${ }^{1}$ Department of Public Health Dentistry, Kothiwal Dental College \& Research Centre, India

Corresponding Author: Pradeep Tangade, Email: ptangade@ rediffmail.com
} 
a similar, but incomplete process, lends support to the dichotomy theory (8). The role of hereditary cannot also be ruled out as inheritance plays a vital role in tooth development.

The effects of supernumerary teeth on the developing dentition may vary. Increased number of erupted teeth, crowding and failure of eruption of adjacent permanent teeth are the most frequent findings associated with it (2). Displacement or ectopic eruption is commonly associated with supernumerary or adjacent teeth. Other related findings can be diastemata, root resorption and loss of vitality of adjacent teeth (7).

\section{CASE REPORT}

A thirteen year old boy (Fig 1) reported to the Department of Public Health Dentistry with the chief complaint of malaligned upper and lower front teeth. The family and medical histories were non contributory. Extra oral examination revealed

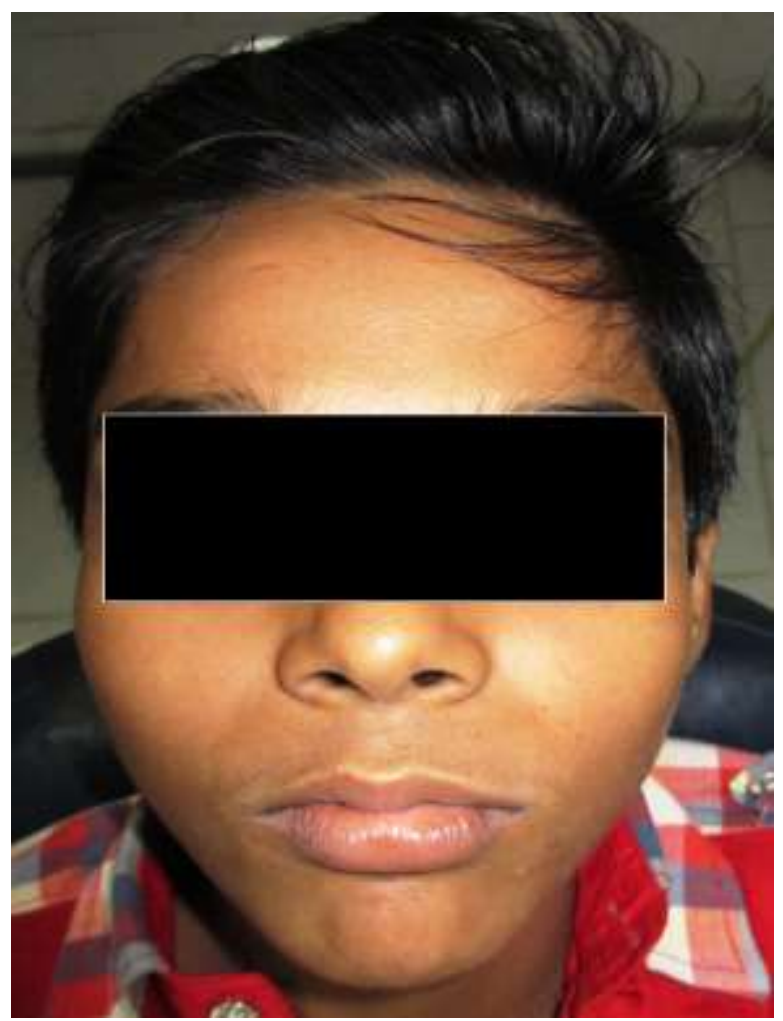

no abnormalities. On intraoral examination, he presented with complete set of permanent dentition in both maxillary and mandibular arches except third molars with presence of supernumerary supplemental maxillary central incisor having morphology similar to that of permanent maxillary central incisors present (Fig $2)$. The supplemental central incisor was rotated because of a discrepancy in arch length. All the three incisors elicited a positive response to thermal and electric pulp testing. The supplemental tooth was figured out on the basis of alignment of the incisors corresponding to mid palatine raphe and facial midline. Therefore, the distal most central incisor on left side was confirmed as supplemental tooth.

The supplemental tooth was similar in size with other maxillary central incisors present in oral cavity. Intra oral peri apical radiograph (IOPA Fig 3) revealed complete root configuration with sound periodontium of central incisor and its supplemental tooth. The crown and root morphology of both left central incisor and supplemental tooth were identical. There was a marked anterior tooth crowding in upper and lower arches. There was no history of trauma nor significant past medical history. To rule out any

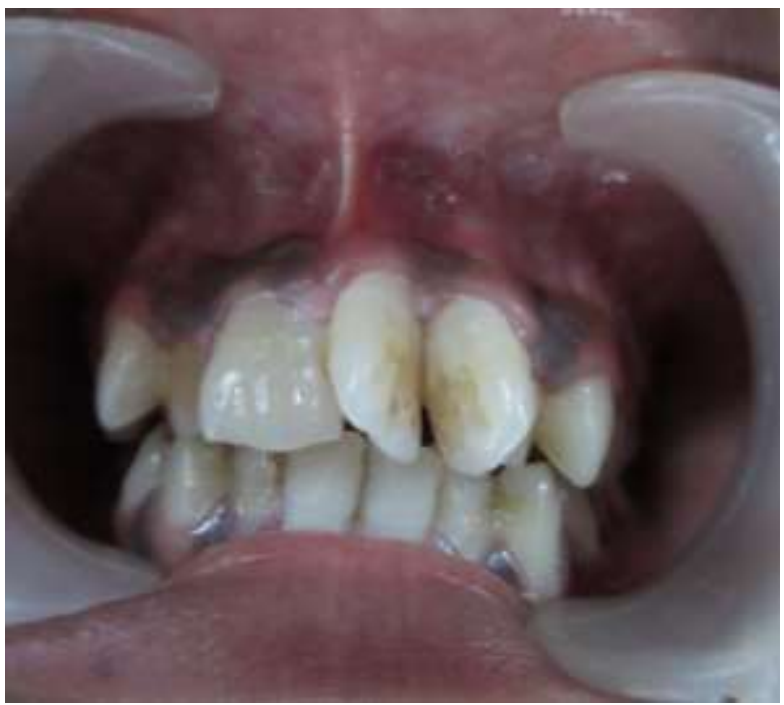

Figure1: Extra oral photograph of patient.

Figure 2: A clinical image showing three central incisors. 
To rule out any associated syndrome with the clinical finding, thorough general examination was done. There were no signs of partial or total absence of the clavicles or open sagittal sutures which ruled out any chances of cleidocranial dysostosis. On further investigations, gardiner syndrome was omitted as diagnosis as there was no multiple adenomatous polyposis of the large intestine or multiple osteomas of the facial bones or any cutaneous epidermoid cysts. Therefore, the patient was diagnosed with non-syndromic unilateral supplemental maxillary incisor. The patient had Angle's class I type I as in both sides the mesio-buccal cusp of the maxillary first molar was aligned directly over the buccal groove of the mandibular first molar and there was crowding in maxillary anterior teeth (Dewey's modification). The teeth present in the mouth were $11,12,13$, $14,25,26,27,21,21 \mathrm{~S}, 22,23,24,25,26,27,31$, $32,33,33,34,35,36,37,41,42,43,44,45,46$, 47.

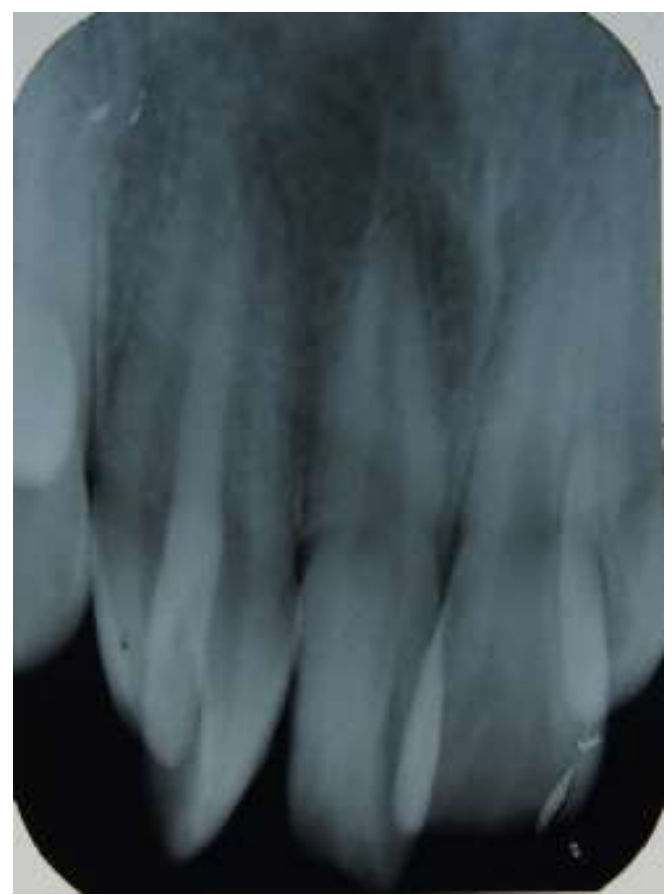

Figure 3: Intra oral periapical radiograph of supplemental tooth.

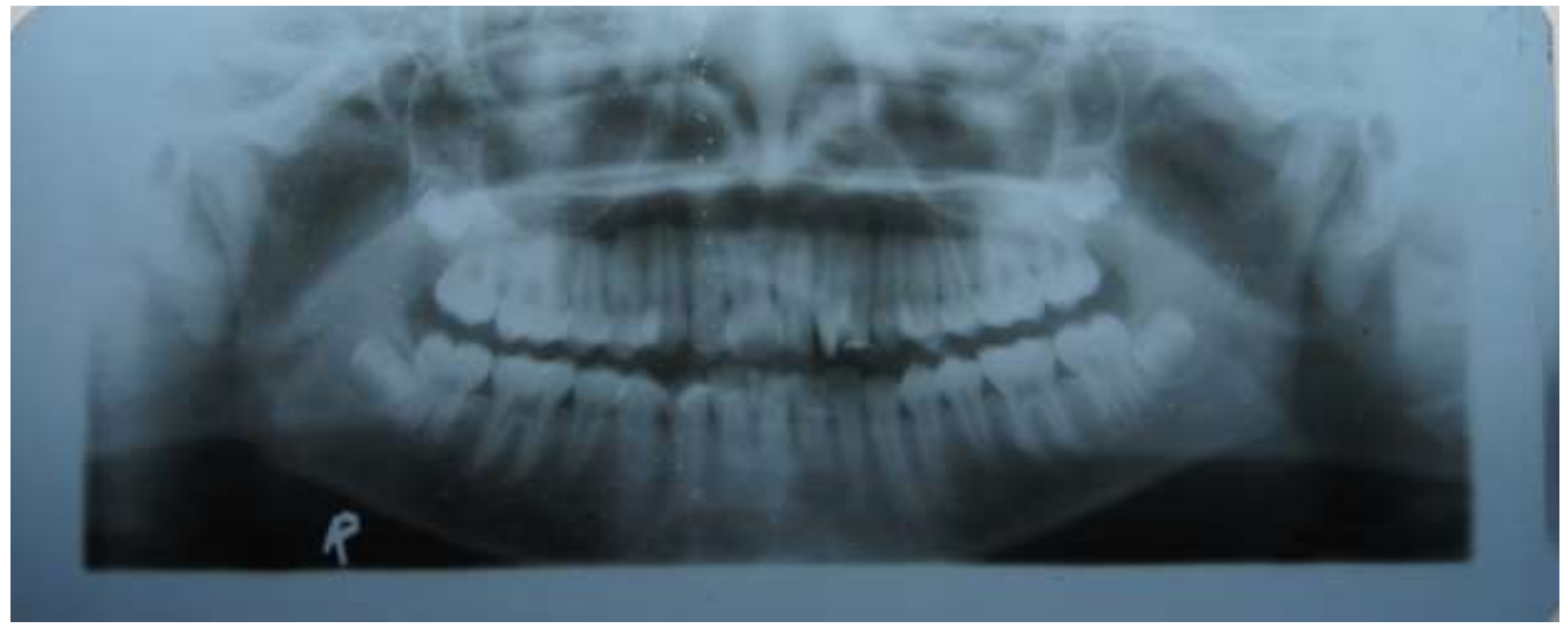

Figure 4: An orthopantomogram showing supplemental maxillary central incisor on left side.

An orthopantomogram (OPG) was taken which revealed complete root configuration with sound periodontium in relation to supplemental The first step in managing supplemental teeth is the correct localization and identification of complications associated with them. The management depends upon the type and position of these teeth and their effects on adjacent teeth. maxillary incisor (Fig 4). The crown and root morphology of supplemental tooth was identical with the other maxillary central incisors present. The patient should be made aware of complications. Considering oral hygiene maintenance as well as aesthetic problems, treatment plan involved extraction of the most distal upper right central incisor, and after this 
patient was advised orthodontic treatment for the correction of crowding in both arches.

\section{DISCUSSION}

The present case is unusual as there are three maxillary central incisors, all the three with normal morphology which is quite rare. Generally most of the supplemental teeth are present bilaterally as seen in previous literature $(9,10)$, but in this is a case of unilateral supplemental tooth.

Most supplemental teeth remain unerupted and being associated with several pathological conditions, such as widened follicular space, dentigerous cyst formation, dental pulp necrosis, pulp canal obliteration, root resorption, and ankylosis (11), but in the present case the supplemental tooth is fully erupted. Disturbance of eruption, diastema formation, and rotations of permanent teeth are common complications.

Timing of surgical removal of supernumerary teeth has been litigious. Hogstrum and Andersson (7) suggested two modes of management. According to the first choice, the supernumerary teeth should be removed as soon as it has been diagnosed, but this could create dental phobia problems for a young child and is also has been further linked with devitalization or deformation of adjacent teeth. Another alternative is to leave the supernumerary teeth till root development of the adjacent teeth is complete. The complications associated with deferring the extraction include loss of eruptive force of adjacent teeth, loss of space and crowding of the affected arch, and midline shifts.

The presence of supplemental teeth of normal morphology is quite a rare condition and is commonly associated with various syndromes but in rare cases italso exists as non-syndromic forms. Supernumerary teeth can cause aesthetic or functional problems, especially when situated in the maxillary anterior region. Complications related to the phenomenon can be minimized by early detection and by carrying out a comprehensive treatment protocol.

\section{REFERENCES}

1. Schulze C. Developmental abnormalities of the teeth and jaws. In: Gorlin RJ, Goldman HM, eds. Thoma's oral pathology. St Louis: CV Mosby, 1970:112-22.

2. Mitchell L. Supernumerary teeth. Dent Update 1989; 16:65-9.

3. Yusof WZ. Non-syndromal multiple supernumerary teeth: literature review. J Can Dent Assoc 1990; 56:147-9.

4. Luten JR. The prevalence of supernumerary teeth in primary and mixed dentitions. J Dent Child 1967; 34:48-9.

5. Rajab LD, Hamdan MA. Supernumerary teeth: review of the literature and a survey of 152 cases. Int J Paediatr Dent 2002; 12, 244-254.

6. Acton CHC. Multiple supernumerary teeth and possible implications. Aust Dent J 1987; 32:48-9.

7. Hogstrum A, Andersson L. Complications related to surgical removal of anterior supernumerary teeth in children. J Dent Child 1987; 54:341-3.

8. Stellzig A, Basdra EK, Komposch G. Mesiodentes: incidence, morphology, etiology. J Orofac Orthop 1997; 58, 144153.

9. Trotman CA, McNamara T. Four maxillary incisors: a case report. Spec Care Dentist 1994;14, 112-115.

10. Camilleri S. A case of bilateral supplemental maxillary central incisors. Int J Paediatr Dent 2003; 13, 57-61.

11. Stafne EC. Supernumerary upper central incisors. Dental Cosmos 1931; 73, 976980. 